\title{
Evaluation of a Kernel Associated with Laminar Flow Tubular Cataly tic Reactors
}

\author{
By Chul-Hee Kim and R. E. Gilbert
}

Introduction. Katz [2] has proposed a method of analysis of tubular catalytic reactors which, in principle, will allow the determination of the actual kinetics of the surface reaction even in the presence of a moderately slow rate of diffusion to and from the surface. The catalyst is deposited on the tube wall and the reacting fluid allowed to flow past under conditions of known hydrodynamics. The reader is referred to the original paper for further details.

A case of particular importance is that for which flow is laminar. For this case, the analysis yields an integral equation involving a kernel

$$
M(\theta)=\left.(\partial H / \partial \theta)\right|_{y=1}
$$

where $H(y, \theta)$ is defined by

$$
\begin{array}{cl}
\frac{\partial}{\partial y}\left(y \frac{\partial H}{\partial y}\right)=4 y\left(1-y^{2}\right) \frac{\partial H}{\partial \theta}, \\
y=0, & H \text { bounded, } \\
y=1, & \partial H / \partial y=1 . \\
\theta=0, & H=0,
\end{array}
$$

The precise evaluation of $M(\theta)$ in a form suitable for computation is the subject of this paper. The variable $\theta$ must assume all positive real values.

Exact Solution. To obtain an exact solution to (2) let

$$
H(y, \theta)=\theta+W(y)+G(y, \theta) \text {. }
$$

Then the differential equation for $H$ can be resolved into the following two problems:

$$
\begin{array}{cl}
\frac{d}{d y}\left(y \frac{d W}{d y}\right)=4 y\left(1-y^{2}\right), \\
y=0, & W \text { bounded }, \\
y=1, & d W / d y=1,
\end{array}
$$

and

$$
\begin{aligned}
\frac{\partial}{\partial y}\left(y-\frac{\partial G}{\partial y}\right)= & 4 y\left(1-y^{2}\right)-\frac{\partial G}{\partial \theta} \\
y=0, & G \text { bounded }, \\
y=1, & \partial G / \partial y=0, \\
\theta=0, & G=-W(y) .
\end{aligned}
$$

Received December 2 $\tau, 1967$. 
These two problems, (4) and (5), along with Eq. (3), are consistent with Eq. (2).

The solution to (4) is

$$
W(y)=B_{0}+y^{2}-y^{4} / 4,
$$

where $B_{0}$ is an integration constant which need not be determined for our purpose.

The solution to (5) may now be obtained by separation of variables as

$$
G(y, \theta)=A_{0}+\sum_{n=1}^{\infty} A_{n} \phi_{n}(y) e^{-\lambda_{n} \theta},
$$

where the constants $A_{n}$ are given by the following ratio of two integrals:

$$
A_{n}=\frac{\int_{0}^{1} 4 y\left(1-y^{2}\right)\left(y^{4} / 4-y^{2}\right) \phi_{n}(y) d y}{\int_{0}^{1} 4 y\left(1-y^{2}\right) \phi_{n}^{2}(y) d y}, \quad n=1,2, \cdots .
$$

The functions $\phi_{n}(y)$ are eigensolutions to the Sturm-Liouville system

$$
\begin{gathered}
\frac{d}{d y}\left(y \frac{d \phi_{n}}{d y}\right)+4 y\left(1-y^{2}\right) \lambda_{n} \phi_{n}=0, \\
y=0, \quad \phi_{n}{ }^{\prime}=0, \\
y=1, \quad \phi_{n}{ }^{\prime}=0 .
\end{gathered}
$$

The first 20 eigenvalues, $\lambda_{n}$, for (9) have been tabulated by Dranoff [1] along with the functions $\phi_{n}(y)$ and the denominator integral of (8). The normalizing condition $\phi_{n}(0)=1$ was chosen for convenience in computation. Using the approach of Sellars. et al. [4], Dranoff also derived some important approximate formulas for $\lambda_{n}$ and $\phi_{n}$ valid for large $n$.

By manipulation of (9) it can be shown [3] that the numerator in (8) is equal to. $-\phi_{n}(1) / \lambda_{n}$. Hence we may write

$$
A_{n}=-\phi_{n}(1) / \lambda_{n} N_{n}
$$

where $N_{n}$ is the normalizing integral appearing in the denominator of (8). Putting. (6), (7), and (8) into (3), we obtain

$$
H(y, \theta)=\theta+B_{0}+y^{2}-\frac{y^{4}}{4}+A_{0}-\sum_{n=1}^{\infty} \frac{\phi_{n}(1)}{\lambda_{n} N_{n}} \phi_{n}(y) e^{-\lambda_{n} \theta}
$$

and applying (1) to Eq. (11) yields the desired kernel

$$
M(\theta)=1+\sum_{n=1}^{\infty} \frac{\phi_{n}^{2}(1)}{N_{n}} e^{-\lambda_{n} \theta} .
$$

Notice that neither $A_{0}$ nor $B_{0}$ appears in (12). This equation is simpler than the expression originally derived by Katz [2] in that it contains only one integral, $N_{n}$.

Numerical Evaluation of the Kernel. Equation (12) requires more and more terms as $\theta$ approaches zero. Using Dranoff's results [1], we were able to get satisfactory convergence of (12) down to about $\theta=0.015$. An asymptotic solution for small $\theta$ is developed in the Appendix. It takes the form

$$
M(\theta) \sim(.256 \cdots) \theta^{-2 / 3} \quad \text { as } \theta \rightarrow 0 .
$$


Unfortunately, Eq. (12) using 20 terms does not give satisfactory agreement with Eq. (13) for the range of $\theta$ near 0.015 . Hence it was necessary to extend the work of Dranoff.

Since Dranoff has supplied approximations to the eigenvalues and eigensolutions for large $n$, the first effort was to use these for values of $n$ above 20. Dranoff's equations yield

$$
\lambda_{n} \sim 4\left(n+\frac{1}{3}\right)^{2}
$$

and

$$
\phi_{n}(1) \sim \frac{(-1)^{n} 3^{1 / 6}}{2^{1 / 3} \Gamma(2 / 3)(3 n+1)^{1 / 3}}=\frac{(-1)^{n} \times .7039 \cdots}{(3 n+1)^{1 / 3}} .
$$

In addition, following Sellars et al. [4], it is easily shown that

$$
N_{n}=-\left.\phi \frac{\partial^{2} \phi}{\partial \lambda \partial y}\right|_{y=1 ; \lambda=\lambda_{n}} \sim \frac{.75}{3 n+1} .
$$

These three approximations are all that are required in (12).

Comparing results from (14), (15), and (16) with actual values at $n=20$ reveals that (16) gives values accurate to about $.02 \%,(14)$ is valid to within $0.1 \%$, but (15) is good only to within about $1.7 \%$. The total error in the quantity $\phi_{n}{ }^{2}(1) / N_{n}$ at $n$ $=20$ is $3.5 \%$ when calculated from (15) and (16). In view of the fact that many terms are required in (12) for small $\theta$, this error was deemed excessive. Hence Dranoff's eigenvalues were extended by direct integration of (9).

Using the Runge-Kutta-Gill integration scheme with double-precision arithmetic on the IBM 360, the eigenvalues of (9) from $n=1$ to $n=41$ were computed. The overall method for extracting these eigenvalues was that described by Dranoff. The calculations at each $n$ were stopped when $\phi_{n}{ }^{\prime}(1)$ was found to be less than $10^{-13}$. These calculations are summarized in Table 1 . The interval of integration for the Runge-Kutta-Gill algorithm is shown in the second column. All results from $n=1$ to $n=20$ agree very closely with those of Dranoff. As a further check on the integration procedure, the last two columns present the numerator integral of Eq. (7) as calculated by direct integration and from its mathematical equivalent, $-\phi_{n}(1) / \lambda_{n}$. Agreement between the two columns is very good.

Using all 41 eigenvalues in Eq. (12), satisfactory convergence was obtained down to about $\theta=.001$. Figure 1 is a plot of $M(\theta)$ on logarithmic coordinates. The asymptote approached for $\theta>.3$ is Eq. (12), using only the first term of the series. The dashed line at the left edge is Eq. (13). It can be seen that $M(\theta)$ is approaching this line asymptotically, although exact agreement is still lacking even at $\theta=.001$.

With these values of the kernel it now becomes possible to test the efficacy of Katz' method for analyzing laminar flow catalytic reactors. The first part of such a program, using computer simulation of a reactor, is now nearing completion.

It has been found that, provided $M(\theta)$ is known accurately down to about .002, only its integral need be known over $0<\theta<.002$ in order to carry out the convolution. The importance of Eq. (13) is then readily seen, as without it there would be no means for estimating such an integral. Furthermore, over this small range of 


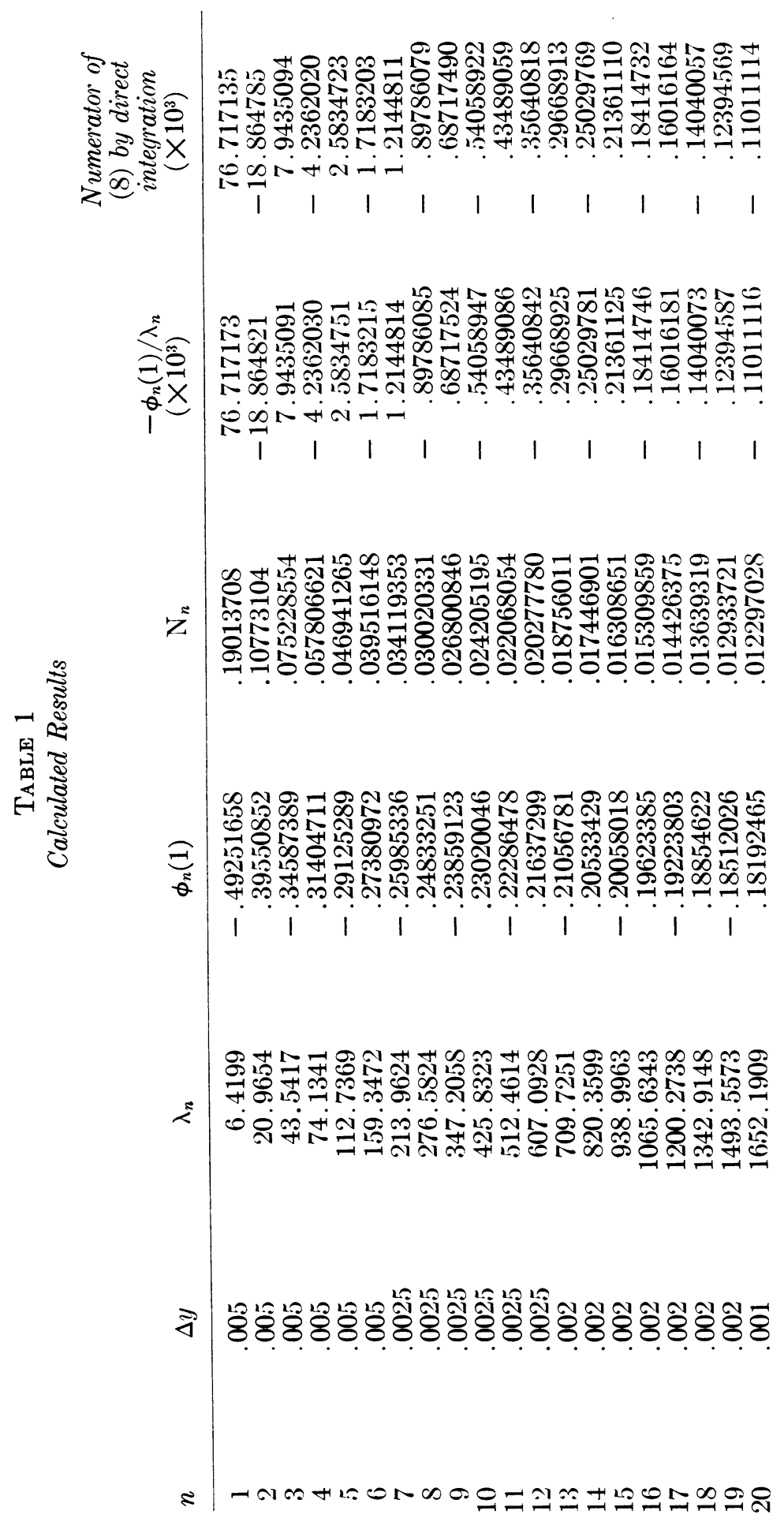




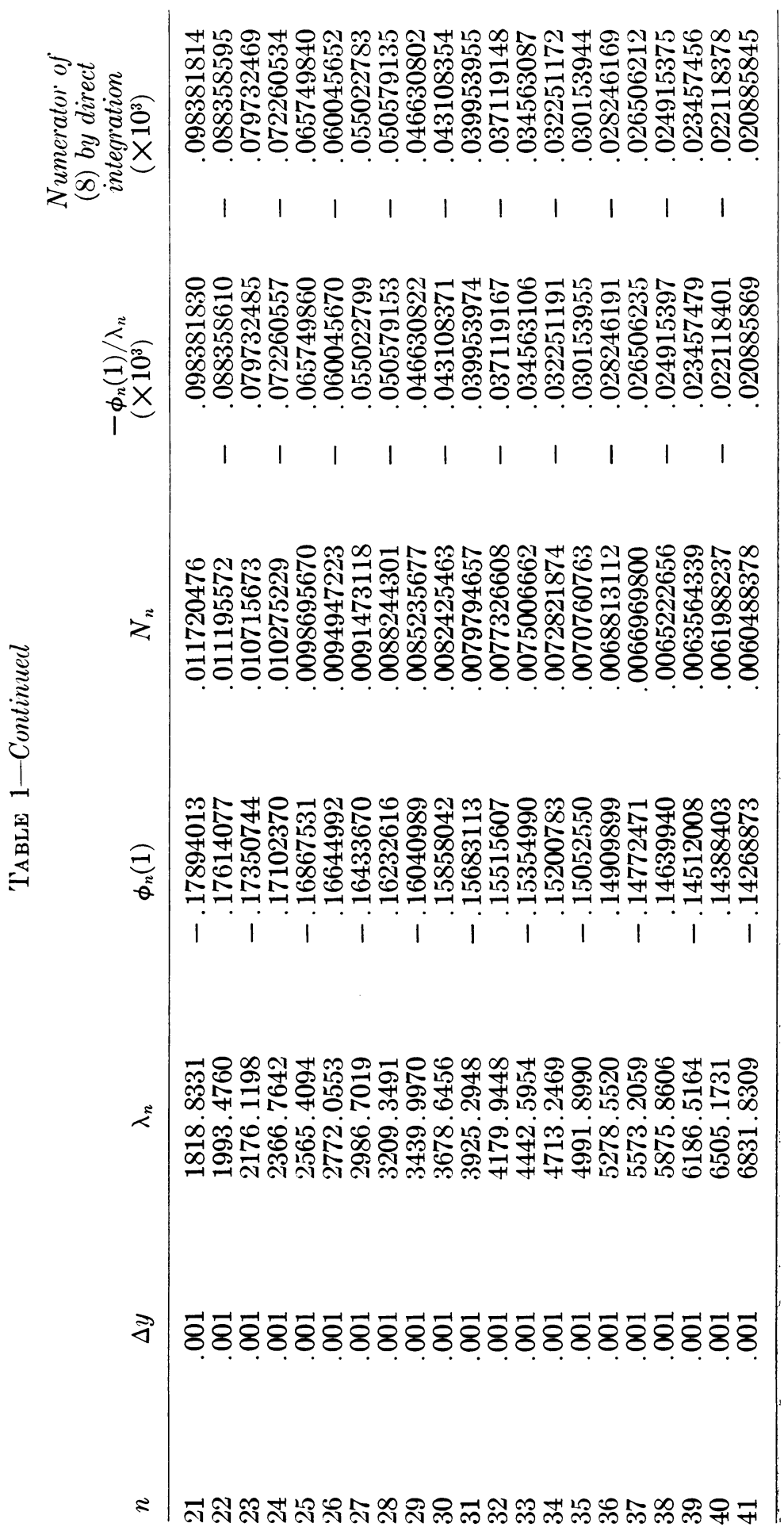


$\theta$ the integral need not be known accurately and so the deviation from the asymptote at the left end of Fig. 1 is not serious.

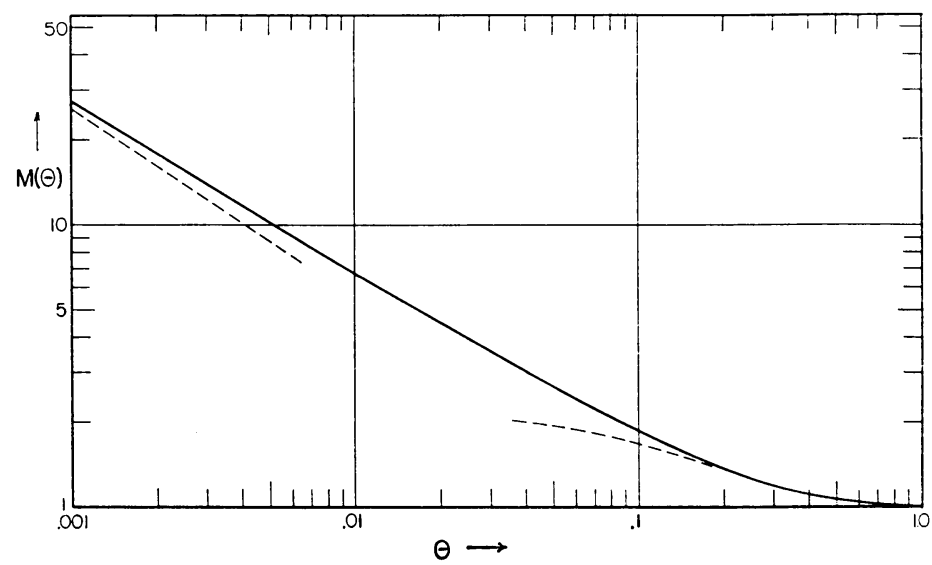

Figure 1. The Kernel $M(\theta)$

\section{ApPendix}

Derivation of the Asymptotic Approximation to $M(\theta)$. We seek a solution to (2) valid for small $\theta$. It is evident from the boundary conditions that the departure of $H$ from its initial condition, $H(y, 0)=0$, will first take place at $y=1$. The disturbance here will then gradually "penetrate" toward $y=0$. This suggests that for small $\theta$ we may substitute $z=1-y$ into (2) and find a solution valid for small $z$. This substitution followed by elimination of higher-order terms in $z$, yields the approximate equation

$$
\partial^{2} H / \partial z^{2}=8 z(\partial H / \partial \theta) .
$$

The Laplace transform of (A1) with respect to $\theta$ is then

$$
d^{2} \bar{H} / d z^{2}-8 s z \bar{H}=0,
$$

which has as a general solution

$$
\bar{H}(z, s)=z^{1 / 2}\left[C_{1} I_{1 / 3}\left(\frac{2(8 s)^{1 / 2}}{3} z^{3 / 2}\right)+C_{2} I_{-1 / 3}\left(\frac{2(8 s)^{1 / 2}}{3} z^{3 / 2}\right)\right] .
$$

For large argument, both Bessel functions in (A2) increase exponentially. To keep $\bar{H}(z, s)$ bounded, it is necessary to set $C_{2}=-C_{1}$. The condition

$$
z=0, \quad d \bar{H} / d z=-1 / s
$$

then yields, after some manipulation of the Bessel functions,

$$
C_{2}=-C_{1}=\frac{\Gamma(1 / 3)}{3^{2 / 3} 2^{1 / 2} s^{7 / 6}} .
$$

From the definition of $M(\theta)$, it is seen that 


$$
\bar{M}(s)=\left.s \bar{H}\right|_{y=1}=\left.s \bar{H}\right|_{z=0} .
$$

Evaluating $\left.\bar{H}\right|_{z=0}$ from (A2) and (A3), inserting the result into (A4), and simplifying, yields

$$
\bar{M}(s)=\frac{0.5 \Gamma(1 / 3)}{3^{1 / 3} \Gamma(2 / 3) s^{1 / 3}},
$$

which, upon inversion and re-arrangement, gives the function

$$
M(\theta)=\frac{1}{3^{4 / 3} \Gamma(5 / 3) \theta^{2 / 3}} .
$$

This is Eq. (13) of the text. Note that although $M(\theta)$ is infinite at $\theta=0$, the area under the curve is finite.

Department of Chemical Engineering

University of Nebraska

Lincoln, Nebraska 68508

1. J. S. DRANOFF, "An eigenvalue problem arising in mass and heat transfer studies," Math. Comp., v. 15 , 1961, pp. 403-409. MR 24 \#A280.

2. S. KatZ, "Chemical reactions catalysed on a tube wall," Chem. Engr. Sci., v. 10, 1959, p. 202.

3. C-H. KIM, A Computer Study of Heterogeneous Laminar Flow Tubular Reactors, M.S. Thesis, Univ. of Nebraska, Lincoln, Nebraska, 1968.

4. J. B. Sellars, M. Tribus \& J. S. Klein, "Heat transfer to laminar flow in a round tube or conduit-the Graetz problem extended," Trans. ASME, v. 78, 1956, p. 441. 